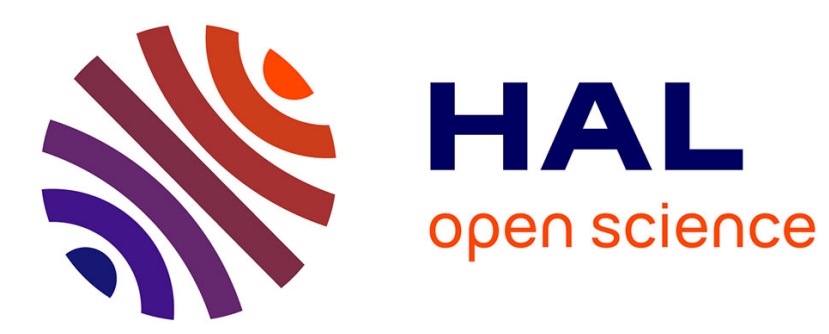

\title{
Inadéquation des qualifications et fracture spatiale
}

\author{
Frédéric Gavrel, Nathalie Georges, Yannick L'Horty, Isabelle Lebon
}

\section{To cite this version:}

Frédéric Gavrel, Nathalie Georges, Yannick L'Horty, Isabelle Lebon. Inadéquation des qualifications et fracture spatiale. Economie et Prévision, 2015, 206-207 (1-2), pp.1-16. 10.3406/ecop.2015.8179 . halshs-01377640

\section{HAL Id: halshs-01377640 \\ https://shs.hal.science/halshs-01377640}

Submitted on 7 Oct 2016

HAL is a multi-disciplinary open access archive for the deposit and dissemination of scientific research documents, whether they are published or not. The documents may come from teaching and research institutions in France or abroad, or from public or private research centers.
L'archive ouverte pluridisciplinaire HAL, est destinée au dépôt et à la diffusion de documents scientifiques de niveau recherche, publiés ou non, émanant des établissements d'enseignement et de recherche français ou étrangers, des laboratoires publics ou privés. 


\title{
Inadéquation des qualifications et fracture spatiale
}

\author{
Frédéric Gavrel ${ }^{(*)}$ \\ Nathalie Georges $^{(* *)}$ \\ Yannick L'Horty ${ }^{(* * *)}$ \\ Isabelle Lebon ${ }^{(* * * *)}$
}

Dans un contexte de chômage élevé, les grandes villes connaissent une ségrégation urbaine qui conduit certains habitants à s'installer dans des zones très éloignées du centre actif de la ville. Tant le manque d'emploi que la fracture spatiale créent des problèmes sociaux tels que les décideurs publics locaux sont amenés à mettre en ouvre des politiques susceptibles de les combattre. Pour étudier analytiquement les effets de ces politiques, nous construisons un modèle d'économie urbaine dans lequel un processus d'appariement avec agents hétérogènes représente le fonctionnement du marché du travail local. En l'absence d'intervention publique, le modèle prédit que la structure du bassin d'emploi exclut les chômeurs des zones de résidence proches des entreprises. Le marché joue ici contre la mixité géographique en conduisant à une ségrégation "parfaite » des habitants en fonction de leur situation sur le marché du travail. À l'équilibre du modèle, on aboutit à un déséquilibre total dans l'espace où les chômeurs sont relégués à l'extrême périphérie. Nous explorons alors les effets des différentes politiques publiques locales permettant de réduire le chômage et la fracture spatiale : politiques de transport, de formation, de logement social, de densification de l'habitat et de fiscalitélocale.

Certains politiques sont efficaces pour réduire le chômage, en abaisser la durée et diminuer les inégalités sociales, telle une baisse des coûts de transport, le développement des actions de formation ou encore, la densification de l'habitat. Mais ces politiques restent sans efficacité contre la fracture spatiale et la ségrégation urbaine. Seule une politique de logement social peut réduire les inégalités spatiales mais ses effets sur le chômage ne sont pas favorables. C'est pourquoi il importe de combiner les instruments de politique publique pour pouvoir combattre à la fois le chômage et la fracture spatiale. Associer la construction de logements sociaux à une politique de formation (qui limite les conséquences de l'inadéquation entre qualifications offertes et demandées) ou au subventionnement des frais de transport permettrait d'atteindre les objectifs visés.

(*) Crem-CNRS (UMR 6211), Université de Caen Normandie et Tepp-CNRS (FR 3435).

E-mail : frederic.gavrel@unicaen.fr

(**) Ensae-Paristech, CEE et Tepp (FR 3435)

E-mail : nathalie.georges@dgtresor.gouv.fr

(***) Université Paris-Est, Erudite (EA 437), UPEC, UPEM, TEPP CNRS (FR 3435), Marne-La-Vallée.

E-mail : Yannick.lhorty@univ-mlv.fr

(****) Crem-CNRS (UMR 6211), Université de Caen Normandie et Tepp-CNRS (FR 3435).

E-mail : isabelle.lebon@unicaen.fr

Les auteurs remercient les deux rapporteurs anonymes pour leurs remarques constructives qui ont grandement contribué à l'amélioration de cet article.

Cet article n'engage que ses auteurs et non les institutions auxquelles ils appartiennent. Il n'engage a fortiori ni la Direction générale du Trésor, ni le ministère des Finances et des Comptes publics, ni le ministère de l'Économie, de l'Industrie et du Numérique. 
Pour financer ces mesures, on peut envisager de prélever un impôt soit sur les entreprises, soit sur les travailleurs. L'imposition des entreprises réduit leur profitabilité et donc le nombre des implantations d'unités productives dans la ville considérée, ce qui va dégrader la situation des travailleurs locaux. Une taxe d'habitation pesant directement sur les ménages semble moins défavorable à l'emploi, si elle est paramétrée de façon à diminuer régulièrement avec la distance au centre. Les recettes fiscales ainsi obtenues pourront être partagées entre l'investissement dans les logements sociaux et par exemple la formation des travailleurs. Une répartition adéquate permettra de créer une véritable réduction de la fracture spatiale sans conséquence négative sur l'état du marché du travail. 
Depuis plusieurs décennies, la répartition de la population dans l'espace urbain s'est imposée en tant qu'objet d'étude. À l'origine, cette thématique s'est nourrie des observations faites sur la structure des villes, et notamment des villes américaines, à la suite de l'article précurseur de Kain (1968). Cet auteur a constaté l'existence dans l'espace urbain d'une ségrégation spatiale qui dans le cas des États-Unis était aussi une ségrégation ethnique. Le centre historique des villes américaines était alors occupé pour l'essentiel par les populations noires défavorisées, tandis que les entreprises se délocalisaient vers les banlieues blanches aisées. Kain a souligné que les noirs restés au centre-ville souffraient d'un taux de chômage structurel élevé, phénomène qu'il attribuait à l'éloignement des emplois.

À partir de ce travail fondateur, s'est développée une vaste littérature tant empirique que théorique dont la finalité était de mesurer et d'expliquer les effets de la localisation des travailleurs sur leurs capacités à retrouver un emploi. Les études empiriques menées sur données américaines semblent confirmer le rôle joué par la distance aux emplois dans la difficulté à sortir du chômage, puisque les travailleurs qui résident à proximité des bassins d'emploi passent en moyenne moins de temps que les autres au chômage, et ce notamment en raison des coûts de prospection induits par la distance aux emplois (Rogers, 1997 ; Immergluk, 1998), ou du coût prohibitif des transports (Brueckner et Martin, 1997). Des résultats comparables ont été obtenus sur données françaises par Cavaco et Lesueur (2004).

Cependant, Hellerstein, Neumark et McInerney (2008) qui étudient la situation de Chicago, montrent que la distance physique à l'emploi importe peu dès lors que l'on prend en compte les problèmes d'inadéquation des qualifications. Des effets de réseaux sont également à considérer pour expliquer le chômage de longue durée de certaines catégories (O'Reagan, 1993). La distance des travailleurs aux emplois ne semble donc pas suffisante pour comprendre le niveau et la durée du chômage. Quant à la répartition des salariés et des chômeurs dans l'espace urbain, on pourrait y voir la conséquence et non la cause de leur statut sur le marché du travail.

C'est la logique qui prévaut dans les modèles usuels d'économie urbaine développés à la suite de l'ouvrage de Fujita (1989). Selon ces modèles, la ségrégation se produit spontanément, car les individus se répartissent dans l'espace urbain en fonction des loyers qu'ils sont disposés à payer. Les habitations les plus prisées sont celles situées au centre-ville où s'établissent les entreprises. Ce sont les coûts de transport payés par les uns et économisés par les autres qui sont à l'origine de la modulation des loyers entre le centre et la périphérie. Les salariés qui ont à la fois le besoin de s'installer à proximité des entreprises, car leur emploi les oblige à s'y rendre très fréquemment, et la capacité financière de payer des loyers élevés aux propriétaires fonciers, choisissent d'habiter à proximité du centre. Il en résulte une véritable fracture spatiale, car les demandeurs d'emploi sont relégués en périphérie urbaine. Si l'on veut sortir de ce résultat standard, il est nécessaire d'introduire des coûts autres que les transports liés à l'éloignement du centre-ville. C'est ainsi que Wasmer et Zenou (2006) montrent comment une meilleure probabilité d'embauche peut conduire certains chômeurs à rester à une faible distance des entreprises malgré l'importance des loyers qu'ils doivent supporter.

L'objectif de notre analyse est complètement différent. Il ne s'agit pas de chercher dans le comportement des travailleurs une explication à l'imperfection de la fracture spatiale telle que l'on peut l'observer dans la réalité, mais d'identifier parmi les politiques publiques susceptibles d'être mises en œuvre localement celles permettant de réduire la fracture spatiale et de conduire à une véritable mixité sociale. Nous verrons notamment si les politiques adéquates consistent à agir directement sur le marché de l'immobilier ou s'il convient plutôt d'intervenir par le biais d'une amélioration du fonctionnement du marché du travail local. De façon plus générale, nous nous interrogeons sur les effets de différents instruments de politiques publiques sur le chômage, la productivité des entreprises et la fracture spatiale. Nous étudions donc des politiques bien plus variées que Zenou (2000) qui se concentre sur la subvention des coûts de transport, puisque nous envisageons également la densification de l'habitat, le logement social et la formation des travailleurs et abordons la question de la capacité de financement de ces mesures par la collectivité locale.

Notre modélisation du marché du travail diffère elle aussi de ce qui est usuellement proposé dans les modèles d'économie urbaine. Nous optons en effet pour une spécification qui puisse refléter les problèmes d'inadéquation des qualifications souvent identifiés comme à l'origine des difficultés de retour à l'emploi. C'est pourquoi nous utilisons un modèle d'appariement dans lequel les agents, travailleurs et entreprises, sont différenciés selon les qualifications offertes par les uns et demandées par les autres. Dans ce cadre, le processus d'embauche est compliqué par l'inadéquation qui peut exister entre les besoins de l'entreprise et les capacités du travailleur (Marimon et Zilibotti, 1999), ce qui affecte directement la productivité et la richesse produite dans l'espace urbain. En fonction des conditions du marché du travail elles-mêmes influencées par les politiques publiques mises en œuvre par les autorités locales, les agents seront plus ou moins exigeants quant à la proximité nécessaire entre les deux partenaires et les entreprises plus ou moins productives (Gavrel et Lebon, 2008).

Dans ce cadre d'analyse, différentes politiques publiques peuvent être envisagées pour améliorer la situation du marché du travail local. Nous montrons 
cependant que les politiques efficaces pour réduire la durée et le volume du chômage restent sans effet sur la fracture spatiale, les chômeurs demeurant cantonnés à la périphérie de la ville. Seule une implication directe du décideur public sur le marché de l'immobilier peut parvenir à modifier la répartition des travailleurs dans l'espace urbain.

La première partie propose un modèle d'appariement avec des entreprises et des travailleurs hétérogènes. Elle explique la répartition des habitants dans l'espace urbain en fonction de leur situation sur le marché du travail et montre comment une fracture spatiale s'instaure entre les chômeurs et les salariés. La deuxième partie présente la résolution du modèle et donne quelques éléments de statique comparative. Les différentes mesures de politique publique visant à réduire la fracture spatiale et le chômage, ainsi que leur financement par des taxes locales, sont étudiées dans la troisième et dernière partie.

\section{Un modèle d'appariement avec fracture spatiale}

Le modèle décrit le fonctionnement du marché du travail sur un bassin d'emploi urbain. Les habitants de ce territoire offrent leur travail aux entreprises situées au centre de la ville. Les travailleurs se distinguent par leur type de qualification, et les emplois par les compétences qu'ils requièrent pour mettre en œuvre la production. Travailleurs et emplois sont ainsi différenciés horizontalement. La productivité de l'emploi dépend de l'importance de l'inadéquation entre la qualification du travailleur et les besoins de l'entreprise (ou skill mismatch).

Pour travailler, tous les salariés doivent se déplacer jusqu'au centre-ville, ils vont donc supporter des coûts de transport d'autant plus élevés qu'ils habitent à une distance importante des entreprises. L'existence de ces coûts de transports explique la modulation des loyers dans l'espace urbain. Les chômeurs dont les revenus sont plus faibles et les besoins en déplacement plus limités que ceux des salariés sont conduits à s'installer à la périphérie de la ville. Ce partage de l'espace urbain produit alors une véritable fracture spatiale.

\section{Différenciation des travailleurs et des emplois et processus d'appariement}

Les travailleurs habitant la ville se distinguent par le type, et non le niveau, de leur qualification.

Les entreprises obtiennent une production maximale de leur unique emploi avec un salarié qui possède des compétences parfaitement adaptées à leurs besoins. Elles peuvent embaucher un travailleur d'un autre type mais au prix d'une perte de productivité qui dépend de l'écart entre les qualifications requises par leur emploi et celles que possède le salarié.

On peut représenter cette différenciation horizontale des qualifications en supposant que les deux types d'agents, travailleurs et entreprises, se répartissent autour d'un cercle virtuel dont chaque point représenterait un type de qualification spécifique. Comme le montrent Marimon et Zilibotti (1999), les qualifications demandées par les entreprises entrant sur le marché du travail s'ajustent aux qualifications disponibles localement afin de maximiser la valeur de leurs emplois vacants. Le cercle des qualifications offertes et celui des qualifications demandées coïncident donc automatiquement. Ce résultat qui peut sembler en contradiction avec la réalité, s'appuie sur l'hypothèse simplificatrice d'entreprises qui toutes produisent un bien indifférencié. Il ne peut donc pas y avoir de qualification considérée comme «inutile » et inutilisable.

Le plus court des deux arcs de cercle séparant un travailleur et un emploi mesure l'inadéquation entre les compétences offertes et les besoins de l'entreprise (voir figure 1). Sans perte de généralité, la circonférence du cercle est supposée égale à 2. L'inadéquation maximale est donc égale à l'unité (Gavrel et Lebon, 2008).

\section{Figure 1 : le cercle des qualifications}

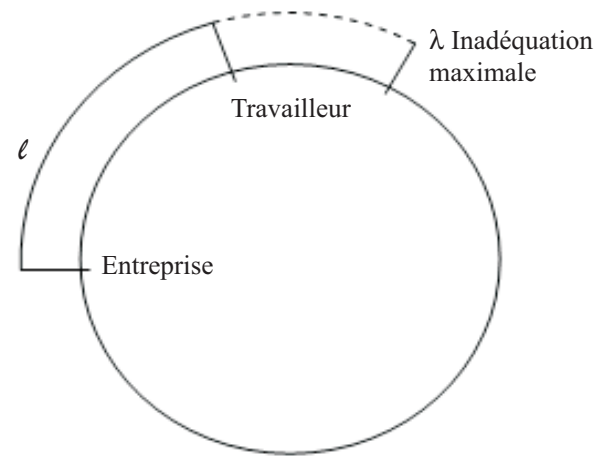

La productivité d'un emploi occupé dépend donc de la distance $\ell$ entre le type de qualification du travailleur et le type de qualification de l'entreprise. $\ell=0$ correspond à la parfaite adéquation des compétences du travailleur au poste, alors que le cas $\ell=1$ correspond au contraire à l'inadéquation maximale possible. En d'autres termes, $\ell$ traduit le degré d'inadéquation des qualifications (ou skill mismatch). Plus $\ell$ est élevé, plus l'inadéquation est forte. La productivité d'un emploi occupé qui décroît avec $\ell$, est définie par $y(\ell)$, c'est une fonction de l'adéquation $\left(y^{\prime}(\ell)<0\right)$. 
Au-delà d'une certaine inadéquation, la productivité potentielle d'un couple entreprise/travailleur n'est plus suffisante pour qu'il leur soit profitable de s'associer. On note $\lambda$ cette inadéquation limite que nous endogénéiserons ultérieurement.

La technologie des contacts entre les entreprises dont l'emploi est vacant et les chômeurs est formalisée par une fonction d'appariement (Pissarides, 2000) et la recherche est supposée non orientée. Selon cette modélisation usuelle, le nombre de contacts, noté $h$, est une fonction croissante des nombres de chômeurs et d'emplois vacants, notés respectivement $u$ et $v(h=h(v, u))$. $h($.$) étant homogène de degré 1$, la part des emplois vacants et des chômeurs entrant en contact avec un partenaire potentiel est une fonction de la tension sur le marché du travail, $\theta(\theta=v / u)$. $q$, la proportion des emplois vacants bénéficiant d'un contact avec un chômeur $(q=h / v=q(\theta))$, est en relation décroissante avec la tension $\theta\left(q^{\prime}(\theta)<0\right)$, alors que $p$, la proportion des chômeurs contactés $(p=h / u=p(\theta)=\theta q(\theta))$, est au contraire en relation croissante avec cette même variable $\left(p^{\prime}(\theta)>0\right)$.

Les contacts ne débouchent pas nécessairement sur une embauche. Comme nous l'avons vu précédemment, si la distance séparant les qualifications de l'emploi et du travailleur est supérieure à l'inadéquation limite $\lambda$, les deux agents n'ont pas intérêt à s'associer et ils choisiront de poursuivre leur recherche afin de trouver un partenaire mieux adapté. Sachant que la recherche est non orientée, la distance qui sépare une entreprise et un emploi vacant a la même probabilité de prendre n'importe quelle valeur entre 0 et 1 . L'inadéquation limite $\lambda$ qui est évidemment comprise dans cet intervalle $(0 \leq \lambda \leq 1)$, mesure la probabilité pour qu'une rencontre entre une entreprise et un travailleur puisse déboucher effectivement sur une embauche.

La part des emplois vacants qui sont finalement pourvus, s'écrit : $\lambda q(\theta)$, alors que la proportion des chômeurs qui trouvent un emploi est égale à : $\lambda p(\theta)$.

L'inadéquation effective entre le type d'un salarié et celui de l'entreprise qui l'emploie va ensuite déterminer l'utilité et le profit intertemporels. La limite $\lambda$ affecte en effet la probabilité de voir aboutir la recherche de l'ensemble des agents qui sont engagés dans le processus d'embauche, elle fixe aussi la productivité moyenne des couples entreprise/salarié qui seront créés.

\section{Utilités et profits intertemporels}

\section{Les entreprises}

L'unique emploi d'une entreprise peut être soit vacant soit occupé. La valeur d'un emploi occupé $J_{F}(\ell)$ dépend de la distance qui sépare la qualification du salarié de la qualification parfaitement adaptée à cet emploi. L'inadéquation $\ell$ va déterminer la productivité effective du couple entreprise/salarié $y(\ell)$, tout comme le salaire négocié $w(\ell)$. En notant $J_{V}$ la valeur d'un emploi vacant et sachant que tous les agents de l'économie escomptent le temps au même taux $r$, on obtient :

(1) $r J_{F}(\ell)=y(\ell)-w(\ell)-s\left[J_{F}(\ell)-J_{V}\right]$

où $s$ est le taux de la loi de Poisson qui régit la destruction des emplois occupés à la suite d'un choc de productivité négatif (Pissarides, 2000). Le cas échéant, la perte en termes de profit intertemporel supportée par l'entreprise, $\left(J_{F}(\ell)-J_{V}\right)$, est d'autant plus forte que l'adéquation des qualifications était bonne.

La valeur d'un emploi vacant est une fonction de l'inadéquation limite $\lambda$ qui agit sur la probabilité de recrutement, tout comme l'espérance de profit d'un poste occupé, notée $\bar{J}_{F}$. Soit $c$ le coût lié à l' annonce du poste vacant (et à son entretien). On a :

(2) $r J_{v}(\lambda)=-c+\lambda q(\theta)\left[\bar{J}_{F}-J_{v}(\lambda)\right]$

où l'espérance $\bar{J}_{F}$ s'écrit :

$\bar{J}_{F}=\frac{1}{\lambda} \int_{0}^{\lambda} J_{F}(\ell) d \ell$

En supposant que la règle de libre-entrée s'applique sur le bassin d'emploi, l'espérance de profit des emplois vacants est nulle $J_{v}(\lambda)=0$. Il en résulte que la combinaison des équations (1) et (2) donne :

(3) $\bar{J}_{F}=\frac{\bar{y}-\bar{w}}{r+s}=\frac{c}{\lambda q}$

où $\bar{y}$ et $\bar{w}$ sont respectivement la production et le salaire moyens sur le bassin d'emploi :

(4) $\bar{y}=\frac{1}{\lambda} \int_{0}^{\lambda} y(\ell) d \ell$ et $\bar{w}=\frac{1}{\lambda} \int_{0}^{\lambda} w(\ell) d \ell$

Suivant l'équation (3), le profit espéré d'un emploi occupé est égal au coût espéré d'un emploi vacant, étant données les durées (moyennes) de ces états.

\section{Les travailleurs}

Comme la productivité des salariés dépend de l'adéquation de leurs compétences à l'emploi occupé, leur salaire $w(\ell)$ en dépend également. L'espérance d'utilité des chômeurs est déterminée par leur opportunité de trouver un emploi et par le salaire moyen qu'ils obtiendront, les deux dépendent notamment de l'inadéquation limite $\lambda$.

En outre, le bien-être des travailleurs est fonction du coût engendré par leur localisation dans l'espace urbain qui est une combinaison du coût de transport qu'ils supportent pour se rendre au centre-ville et du 
loyer qu'ils paient au propriétaire foncier. Soit $x$ la distance entre le lieu d'habitation d'un travailleur et le centre-ville qui est le point 0 où sont localisées l'ensemble des entreprises. Dans un premier temps, nous noterons $T_{E}(x)$ et $T_{U}(x)$ les coûts de localisation supportés respectivement par un salarié et par un chômeur, ces coûts seront explicités ultérieurement.

Soit $V_{E}(\ell, x)$ et $V_{U}(\lambda, x)$ les utilités intertemporelles d'un salarié et d'un chômeur habitant à une distance du centre-ville, on a :

$$
\begin{aligned}
r V_{E}(\ell, x) & =w(\ell)-T_{E}(x) \\
& -s\left[V_{E}(\ell, x)-V_{U}(\lambda, x)\right]
\end{aligned}
$$

L'espérance du chômeur en cas d'embauche est égale à l'utilité moyenne d'un salarié. Soit $d$ la distance entre le centre et la périphérie de la ville, c'est donc potentiellement la distance maximale entre la localisation des entreprises et celle des travailleurs. $\bar{V}_{E}$ se calcule comme :

$\bar{V}_{E}=\frac{1}{\lambda} \frac{1}{d} \int_{0}^{\lambda} \int_{0}^{d} V_{E}(\ell, x) d \ell d x$

L'utilité intertemporelle du chômeur s'écrit quant à elle :

(6) $r V_{U}(\lambda, x)=b-T_{U}(x)+\lambda p(\theta)\left[\bar{V}_{E}-V_{U}(\lambda, x)\right]$

où $b$ représente les allocations-chômage.

\section{Marché de l'immobilier, coûts de transport et fracture spatiale}

Dans un souci de simplicité, nous avons supposé que l'ensemble des entreprises qui se localisent au centre-ville (point 0, dit «Central Business District» (CBD) ou quartier des affaires du centre-ville dans la littérature), occupent un espace nul. À l'inverse, chaque travailleur occupe un espace de taille identique (Zenou, 2000 et Wasmer et Zenou, 2006), pour lequel il doit payer un loyer au propriétaire. Pour réduire leurs coûts de transport, les travailleurs souhaitent habiter au plus près des entreprises. La taille de la ville, et donc la distance du centre-ville à la périphérie $d$, est déterminée par le nombre de ses habitants, supposé donné, et la surface unitaire dévolue à chacun.

On note $R(x)$ le loyer payé par un travailleur qui habite à une distance $x$ du centre-ville (pour $0 \leq x \leq d)$. Les coûts de transports payés par ce travailleur varient suivant qu'il est salarié ou chômeur, car un salarié se déplace plus souvent pour travailler que ne le fait un chômeur pour mener à bien sa recherche d'emploi. Plus précisément, pour une même distance $x$, le salarié assume un coût de transport égal à $t x$, alors que celui qu'il aurait à supporter en tant que chômeur serait réduit à $\alpha t x$ (avec $0<\alpha<1$ ).

Le coût global entraîné par la localisation spatiale s'écrit alors, respectivement pour un salarié et un chômeur :

(7) $T_{E}(x)=R(x)+t x$

(8) $T_{U}(x)=R(x)+\alpha t x$

Les travailleurs quelle que soit leur situation cherchent à minimiser ce coût global, afin de maximiser leur espérance d'utilité(1). C'est ce comportement qui va déterminer les loyers sur l'ensemble du territoire. Le marché immobilier ne peut en effet avoir atteint une situation d'équilibre si certains habitants de la ville sont susceptibles d'améliorer leur utilité en déménageant en dehors ou à l'intérieur de l'espace urbain, alors même que leur situation sur le marché du travail n'a pas changé.

L'équilibre du modèle implique donc que les travailleurs résidant à la périphérie de la ville ne soient pas tentés de franchir les limites de celle-ci pour réaliser une économie sur leur loyer, au prix d'une hausse infinitésimale de leur coût de transport. Le loyer à la périphérie de la ville doit donc être identique à celui du territoire contigu (Fujita, 1989, Wasmer et Zenou, 2006). Le prix de location du terrain à l'extérieur de la ville, noté $R_{A}\left(\operatorname{avec} R_{A} \geq 0\right)$, peut représenter par exemple le loyer de la terre agricole.

Quels suppléments de loyer les travailleurs sont-ils prêts à accepter pour se rapprocher du centre-ville et des entreprises?

En minimisant les coûts de localisation définis par les équations (7) et (8), il apparaît que :

(9) $\min T_{E}(x) \Leftrightarrow R^{\prime}(x)=-t$

(10) $\min T_{U}(x) \Leftrightarrow R^{\prime}(x)=-\alpha t$

Un salarié minimise ses coûts de localisation (et donc maximise son utilité relativement à sa situation géographique) en différents points de la ville à condition qu'un éloignement du centre d'une distance égale à 1 lui permette d'économiser un montant de loyer égal à $t$. Un chômeur est prêt à faire le même déménagement pour une baisse de loyer réduite à $\alpha t$, du fait d'une fréquence de déplacement plus faible.

Cette différence de coût de transport à distance égale conduit les chômeurs à délaisser le centre de la ville où la disponibilité des salariés à payer pour la proximité détermine des niveaux de loyer tels que les chômeurs améliorent leur utilité en s'installant en 
Figure 2 : structuration de l'espace
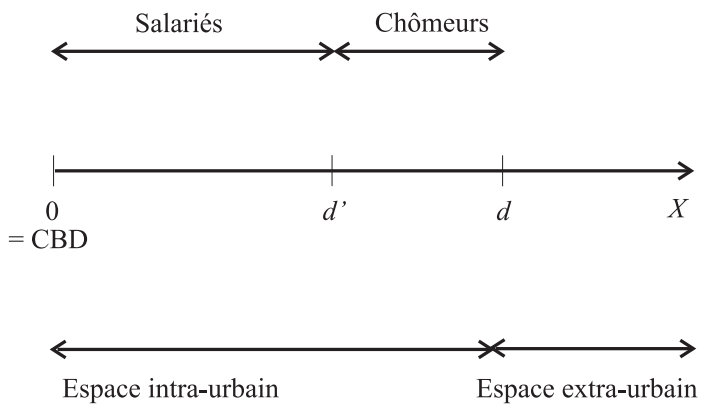

périphérie (voir figure 2). Une fracture spatiale entre ceux qui ont un emploi et ceux qui en sont privés se met donc en place, car les salariés s'installent à une distance des entreprises comprise entre 0 et $d^{\prime}$, alors que les chômeurs se trouvent « repoussés » entre $d^{\prime}$ et $d$.

La distance $d$ ' est endogène et déterminée par le taux de chômage existant dans l'agglomération, noté $u$ (en supposant la population normalisée à 1). On a en effet :

(11) $d^{\prime}=(1-u) d$

Une fracture spatiale aussi nette suppose évidemment que les travailleurs puissent déménager, immédiatement et sans coût, lorsqu'ils perdent leur travail ou à l'inverse en trouvent un. Nous retenons cette hypothèse usuelle des modèles d'économie spatiale.

À l'intérieur de chaque espace, celui qu'habitent les salariés et celui où s'installent les chômeurs, l'équilibre du marché de l'immobilier impose également qu'aucun agent ne puisse améliorer son bien-être en déménageant. C'est cette condition qui va déterminer l'ensemble des loyers en fonction de la distance au point 0 .

Nous repartons du loyer $R_{A}$ payé par les chômeurs qui habitent tout à fait à la périphérie de la ville, à une distance $d$ des entreprises. Un chômeur installé à la distance $d$ paie le loyer le plus faible $\left(R_{A}\right)$ et le coût de transport le plus élevé $(\alpha t d)$. Pour qu' aucun chômeur ne soit tenté de déménager, il faut que le coût global soit le même pour tous $\left(T_{U}(x)=T_{U}, \forall x \in\left[d^{\prime}, d\right]\right)^{(2)}$ :

(12) $T_{U}=R_{A}+\alpha t d=R(x)+\alpha t x$

(pour $d^{\prime} \leq x \leq d$ )

Puisque le coût de la localisation est identique pour tous les chômeurs, tous ont aussi la même utilité intertemporelle.

On en déduit le montant des loyers pour les distances comprises entre $d^{\prime}$ et $d$ :

(13) $R(x)=R_{A}+\alpha t(d-x)$

(pour $d^{\prime} \leq x \leq d$ )
Étant données les équations (11) et (13), le chômeur habitant au plus près des entreprises (à la distance $d^{\prime}$ ) paie donc un loyer égal à :

(14) $R\left(d^{\prime}\right)=R_{A}+\alpha t u d$

$R\left(d^{\prime}\right)$ est donc également le coût de location supporté par le salarié le plus éloigné des entreprises. Pour qu'aucun travailleur ne souhaite déménager, il faut que toutes les distances comprises entre 0 et $d^{\prime}$ conduisent au même coût global $\left(T_{E}(x)=T_{E}, \forall x \in\left[0, d^{\prime}\right]\right)$ que celui supporté par ce salarié, d'où :

$$
\begin{aligned}
T_{E} & =R\left(d^{\prime}\right)+t d^{\prime} \\
& =R_{A}+\alpha t u d+t(1-u) d=R(x)+t x
\end{aligned}
$$

(pour $0 \leq x \leq d^{\prime}$ )

On en déduit les loyers aux distances comprises entre 0 et $d^{\prime}$ :

(16) $R(x)=R_{A}+\alpha t u d+t[(1-u)-x]$

(pour $0 \leq x \leq d^{\prime}$ )

L'ensemble des loyers payés aux différentes distances du centre-ville est représenté (en gras) sur la figure 3 .

Figure 3 : les loyers dans l'espace urbain

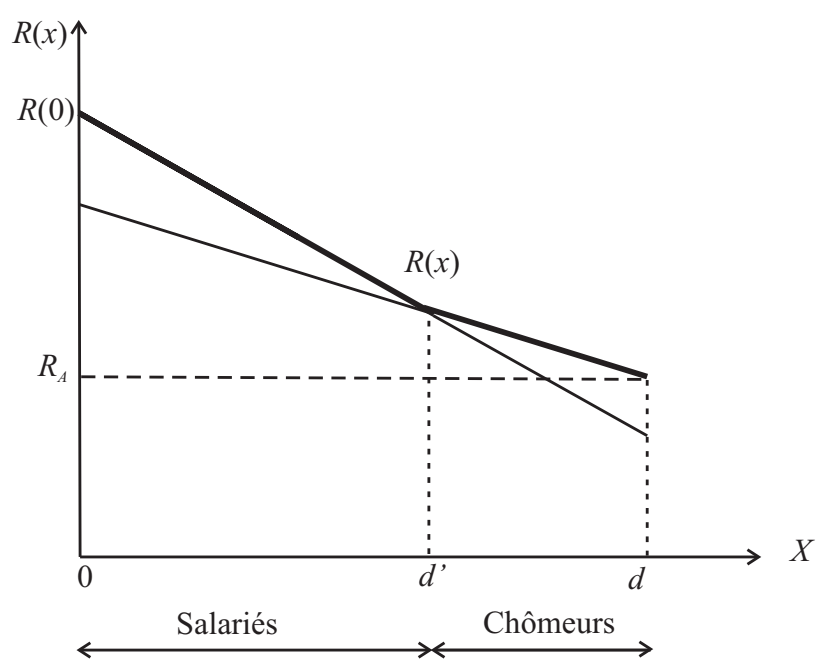

Il apparaît clairement que les loyers augmentent au fur et à mesure que l'on se rapproche du centre-ville, mais cette hausse est plus rapide dans le secteur occupé par les salariés que dans le territoire où s'installent les chômeurs.

Le loyer le plus élevé de la ville, celui qu' acquitte le salarié habitant au point 0 , s'élève donc à :

(17) $R(0)=R_{A}+t d[1-u(1-\alpha)]$

Les loyers du centre-ville sont donc en relation croissante avec la taille de la ville, mesurée par $d$, et 
par conséquent avec la taille de la population qui s'y installe. Ils diminuent en revanche lorsque le taux de chômage s'accroît.

À l'équilibre, $T_{E}$ et $T_{U}$ sont indépendants de la distance à laquelle habitent le salarié et le chômeur. Les espérances d'utilité des travailleurs ne dépendent donc pas de $x$. Le coût de localisation des salariés est supérieur à celui du chômeur $\left(T_{E}>T_{U}\right)$. En effet, à la distance $d^{\prime}$ à laquelle cohabitent salariés et chômeurs, tous payent le même loyer, mais les salariés supportent des coûts de transport plus élevés.

$T_{U}$ est indépendant du taux de chômage. En revanche, le coût de localisation des salariés, $T_{E}$, diminue lorsque le taux de chômage s'accroît. Une hausse du taux de chômage conduit donc à une baisse de la différence entre les coûts de localisation du salarié et du chômeur.

\section{Négociation salariale et inadéquation limite entre travailleurs et emplois}

Un contact entre un chômeur et un emploi vacant ne débouche sur une embauche que si la productivité du couple est suffisante pour engendrer un surplus positif, sinon les deux partenaires ont intérêt à poursuivre leur recherche jusqu'à trouver un partenaire mieux adapté à leur propre type (Marimon et Zilibotti, 1999, Gavrel et Lebon, 2008). L'inadéquation maximale entre la qualification du travailleur et les besoins de l'entreprise est donc celle pour laquelle le surplus est nul :

(18) $V_{E}(\lambda)-V_{U}(\lambda)+J_{F}(\lambda)-J_{V}(\lambda)=0$

La négociation salariale conduit à une répartition du surplus dans des proportions égales respectivement à $\beta$ et $(1-\beta)$, respectivement pour le salarié et l'entreprise (Pissarides, 2000) :

$$
\text { (19) } \begin{aligned}
V_{E}(\ell)-V_{U}(\lambda)= & \beta\left[V_{E}(\ell)-V_{U}(\lambda)\right. \\
& \left.+J_{F}(\ell)-J_{V}(\lambda)\right] \\
\text { (20) } J_{F}(\ell)-J_{V}(\lambda)= & (1-\beta)\left[V_{E}(\ell)-V_{U}(\lambda)\right. \\
& \left.+J_{F}(\ell)-J_{V}(\lambda)\right]
\end{aligned}
$$

En cas d'appariement avec un partenaire dont l'inadéquation est égale à $\lambda$, le surplus de chacun est donc nul. La valeur de l'emploi occupé est donc identique à celle de l'emploi vacant :

(21) $J_{F}(\lambda)-J_{V}(\lambda)=0$

La combinaison des équations (1) et (21) nous donne:

(22) $y(\lambda)=w(\lambda)$
Avec une inadéquation maximale des qualifications, le salaire absorbe donc la totalité de la production du couple entreprise/salarié.

Dans ces conditions, les équations (18) et (19) montrent que l'utilité du salarié est la même que celle d'un chômeur :

(23) $V_{E}(\lambda)=V_{U}(\lambda)$

À partir de (5), (6) et (23), l'on peut écrire :

(24) $w(\lambda)-T_{E}=b-T_{U}+\lambda p(\theta)\left(\bar{V}_{E}-V_{U}(\lambda)\right)$

L'expression (24) va nous permettre de déterminer la valeur de la productivité limite $y(\lambda)$ (égale à celle à celle du salaire $w(\lambda)$ selon (22)). Pour cela nous devons connaître :

- La rente moyenne du salarié par rapport au chômeur, $\left(\bar{V}_{E}-V_{U}(\lambda)\right)$. L'équation (19) permet d'établir que :

$\bar{V}_{E}-V_{U}(\lambda)=\frac{\beta}{1-\beta} \bar{J}_{F}$

Connaissant l'expression de $\bar{J}_{F}$ définie par l'équation (3), on peut écrire :

(25) $\bar{V}_{E}-V_{U}(\lambda)=\frac{\beta}{1-\beta} \frac{c}{\lambda q(\theta)}$

- La différence entre les coûts de localisation du salarié et du chômeur, $\left(T_{E}-T_{U}\right)$. À partir de (12) et (15), on obtient :

(26) $T_{E}-T_{U}=(1-\alpha) t d(1-u)$

La combinaison des équations (22), (24), (25) et (26) donne finalement :

(27) $y(\lambda)=b+(1-\alpha) t d(1-u)+\frac{\beta \theta c}{(1-\beta)}$

La relation (25) définit l'inadéquation limite $\lambda$. Parce qu'elle dépend de la différence entre le coût de localisation payé par le salarié et celui supporté par le chômeur, l'inadéquation limite est ici une fonction du taux de chômage dont nous préciserons l'expression ultérieurement.

\section{Création d'emplois}

La création d'emplois vacants, et donc la tension sur le marché du travail, sont déterminées par le principe de libre-entrée des entreprises sur le bassin d'emploi $\left(J_{V}(\lambda)=0\right)$. 
Pour obtenir facilement l'équation définissant la tension sur le marché su travail, on utilise le surplus moyen, $\bar{S}$ :

(28) $\bar{S}=\frac{1}{\lambda} \int_{0}^{\lambda}\left[V_{E}(\ell)-V_{U}(\lambda)+J_{F}(\ell)-J_{V}(\lambda)\right] d \ell$

Sachant que la répartition du surplus issu de la négociation salariale (équation (20)) s'applique au surplus moyen, on peut écrire :

(29) $(r+s) \bar{J}_{F}=(r+s)(1-\beta) \bar{S}$

On peut également passer par $\bar{S}$ pour décrire la situation du travailleur dont l'espérance d'utilité est égale à la moyenne, et celle du chômeur. À partir des équations (5) et (6), étant donné que l'on a montré que les coûts de localisation sont identiques respectivement pour tous les salariés $\left(T_{E}\right)$ et pour tous les chômeurs $\left(T_{U}\right)$, on a :

(30) $r \bar{V}_{E}=\bar{w}-T_{E}-s\left[\bar{V}_{E}-V_{U}(\lambda)\right]$

(31) $r V_{U}(\lambda)=b-T_{U}+\lambda p(\theta)\left[\bar{V}_{E}-V_{U}(\lambda)\right]$

Si les salariés ont des niveaux d'utilité différents en fonction de leur niveau d'inadéquation avec leur emploi, les chômeurs qui ont tous le même revenu et les mêmes dépenses à la période présente, ainsi que des perspectives semblables, ont tous une utilité identique.

La différence des relations (30) et (31) nous donne:

$$
\begin{aligned}
(r+s)\left(\bar{V}_{E}-V_{U}(\lambda)\right) & =\bar{w}-b-T_{E}+T_{U} \\
& \left.-\lambda p(\theta) \beta\left(\bar{V}_{E}-V_{U}(\lambda)\right)\right]
\end{aligned}
$$

Soit en tenant compte des relations (19) et (28) :

$$
\text { (33) } \begin{aligned}
(r+s)\left(\bar{V}_{E}-V_{U}(\lambda)\right) & =\bar{w}-b-T_{E}+T_{U} \\
& -\lambda p(\theta) \beta \bar{S}
\end{aligned}
$$

Le surplus moyen se déduit finalement de la somme de (29) et (33) :

$(r+s) \bar{S}=\bar{y}-b-\left(T_{E}-T_{U}\right)-\lambda p(\theta) \beta \bar{S}$

ou encore :

(34) $\bar{S}=\frac{\bar{y}-b\left(T_{E}-T_{U}\right)}{(r+s+\lambda p(\theta) \beta)}$

Les relations (3) et (29) nous donnent une seconde expression du surplus moyen :

$$
\text { (35) } \bar{S}=\frac{c}{(1-\beta) \lambda q(\theta)}
$$

En combinant (26), (34) et (35), on peut finalement en déduire la relation qui détermine la tension sur le marché du travail en fonction de l'inadéquation limite :

(36) $(1-\beta) \lambda q(\theta)(\bar{y}-y(\lambda))=c(r+s)$

L'équation (36) qui constitue une des deux relations permettant de définir l'équilibre stationnaire du modèle, sera commentée au paragraphe suivant.

\section{Détermination et analyse de l'équilibre stationnaire}

\section{Équilibre des flux et taux de chômage}

À l'équilibre stationnaire, le flux des travailleurs qui trouvent un emploi est égal au flux des travailleurs qui perdent le leur. Ces flux sont aussi ceux des déménagements intra-urbains. En effet, tous les travailleurs qui perdent leur emploi, améliorent leur utilité en s'éloignant du centre-ville pour mener leur recherche d' un nouvel emploi ; alors que ceux qui en obtiennent un se rapprochent des entreprises, car la hausse du loyer est inférieure aux coûts de déplacement qu'ils vont économiser. Evidemment, cette simultanéité parfaite du changement de statut sur le marché du travail et du changement de lieu d'habitation ne tient qu'à l'absence totale de coût de relocalisation.

Comme la population est normalisée à 1, et en notant $u$ le taux de chômage, la condition d'équilibre des flux s'écrit :

(37) $\lambda p(\theta) u=s(1-u)$

soit :

(38) $u=\frac{s}{s+\lambda p(\theta)}$

Le taux de chômage augmente avec le taux de séparation, $s$, et diminue avec l'inadéquation limite et la tension sur le marché du travail.

\section{Tension sur le marché du travail et inadéquation limite d'équilibre}

La première des deux équations d'équilibre du modèle se déduit de (27) et (38) :

(39) $y(\lambda)=b+(1-\alpha) t d \frac{\lambda p(\theta)}{s+\lambda p(\theta)}+\frac{\beta \theta c}{(1-\beta)}$

Cette relation fait apparaître l'inadéquation limite comme une fonction décroissante de la tension sur le marché du travail, elle est représentée par la courbe $M L$ sur la figure 4. 
Figure 4 : inadéquation limite et tension sur le marché du travail à l'équilibre stationnaire

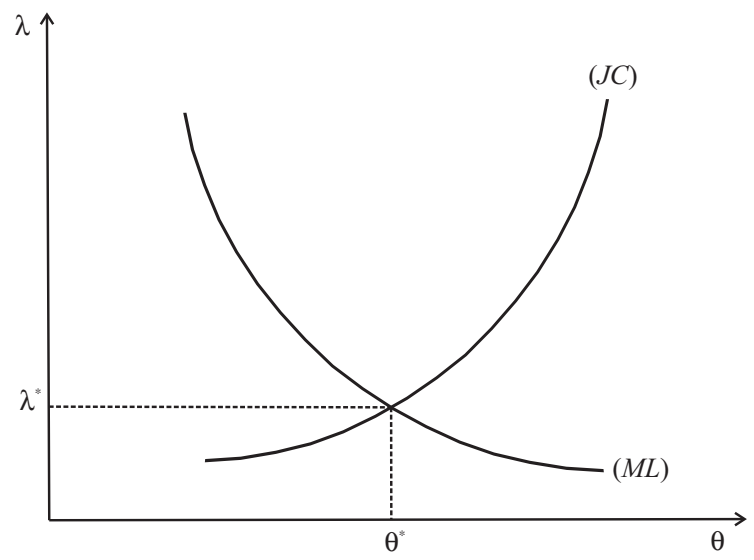

Démonstration. En différentiant (34) en fonction de $\lambda$ et $\operatorname{de} \theta$ nous obtenons :

$$
\begin{aligned}
\partial \lambda \underbrace{\left[y^{\prime}-(1-\alpha) t d \frac{s p(\theta)}{(s+\lambda p(\theta))^{2}}\right]}_{<0} \\
=\partial \theta[\underbrace{\left[(1-\alpha) t d \frac{s \lambda p^{\prime}}{(s+\lambda p(\theta))^{2}}+\frac{\beta c}{(1-\beta)}\right]}_{>0}
\end{aligned}
$$

On en déduit sans ambiguïté $: \frac{\partial \lambda}{\partial \theta}<0$.

Interprétation. La hausse de $\theta$ accroît l'utilité du chômeur en augmentant la probabilité de retour à l'emploi, ainsi que le différentiel de coût de localisation, les appariements devant être de meilleures qualités pour dégager un surplus positif, $\lambda$ doit donc diminuer.

La seconde équation d'équilibre du modèle est celle qui détermine le comportement de création d'emplois vacants (équation (36)). Cette relation définit la tension sur le marché du travail comme une fonction croissante de l'inadéquation limite, elle est représentée par la courbe $J C$ sur la figure 4.

Démonstration. Pour appréhender plus aisément la forme de la relation (36), on réécrit celle-ci en remplaçant $\bar{y}$ par son expression donnée dans l'équation (4):

$$
(1-\beta) q(\theta)\left(\int_{0}^{\lambda} y(\ell) d \ell-\lambda y(\lambda)\right)=c(r+s)
$$

En différentiant l'équation précédente en fonction de $\theta$ et de $\lambda$, on a :

$$
\begin{array}{r}
\partial \underbrace{\left[(1-\beta) q^{\prime}\left(\int_{0}^{\lambda} y(\ell) d \ell-\lambda y(\lambda)\right)\right]}_{<0} \\
=\partial \lambda \underbrace{\left[(1-\beta) q(\theta) \lambda y^{\prime}\right]}_{<0}
\end{array}
$$

D'où : $\frac{\partial \theta}{\partial \lambda}>0$.

Interprétation. La hausse du seuil $\lambda$ multiplie le nombre des contacts qui vont aboutir à une embauche, réduisant ainsi le temps de vacance des emplois. Malgré une certaine détérioration de la productivité moyenne des salariés, le premier effet l'emporte et il en résulte un accroissement de la création d'emplois.

Les équations (36) et (39) déterminent les valeurs d'équilibre de la tension sur le marché du travail et de l'inadéquation limite $\left(\theta^{*}, \lambda^{*}\right)$. À partir de $\theta^{*}$ et de $\lambda^{*}$, on peut obtenir les valeurs d'équilibre des autres variables concernant la ville considérée, notamment le taux de chômage et les loyers.

\section{Quelques éléments de statique comparative}

Nous allons nous intéresser ici à quelques-unes des variables exogènes sur lesquelles les politiques publiques que nous envisagerons dans la section suivante, ne pourront pas porter puisqu'elles ne relèvent pas de la politique locale. Il s'agit d'envisager les effets d'une variation du loyer de la terre à l'extérieur de l'espace urbain $\left(R_{A}\right)$ et d'une évolution du niveau des allocations-chômage $(b)$.

Le prix du terrain à l' extérieur de la ville sur lequel se cale l'ensemble des loyers n'a aucune influence sur le fonctionnement du marché du travail urbain. Cependant, ce résultat est obtenu sous l'hypothèse que les entreprises ne paient pas de loyer. Dans le cas inverse, la hausse de $R_{A}$ qui provoque la hausse des loyers au centre-ville, entraînerait une baisse de leur profitabilité et de la création d'emplois.

La hausse du niveau des allocations-chômage améliore l'utilité du chômeur, mais elle réduit le surplus de l'ensemble des couples productifs

Tableau 1 : statique comparative : hausse des allocations-chômage et du loyer de la terre à l'extérieur de la ville

\begin{tabular}{|l|c|c|c|c|c|c|}
\hline & $\theta$ & $\lambda$ & $u$ & $R(0)$ & $d^{\prime}$ & $R\left(d^{\prime}\right)$ \\
\hline Hausse $R_{A}$ & 0 & 0 & 0 & + & 0 & + \\
Hausse $b$ & - & - & + & - & - & + \\
\hline
\end{tabular}


entreprise/salarié. Le surplus devient donc nul pour une plus faible inadéquation des qualifications, une plus grande exigence est donc nécessaire sur la qualité de l'appariement. La valeur de $\lambda$ doit ainsi diminuer. La hausse de $b$ provoque également la hausse des salaires négociés, réduisant ainsi la profitabilité des entreprises, moins d'emplois vacants sont donc créés, d'où une baisse de $\theta$. Les effets sur l'inadéquation limite et la tension sur le marché du travail se combinent pour provoquer un accroissement du taux de chômage dans l'espace urbain. Il en résulte une diminution de la part du territoire occupé par les salariés et une baisse des loyers au centre-ville.

\section{Les politiques publiques de lutte contre le chômage et la fracture spatiale}

En l'absence d'intervention publique, le modèle prédit que la structure du bassin d'emploi exclut les chômeurs des zones de résidence proches des entreprises. Le marché joue ici contre la mixité géographique en conduisant à une ségrégation «parfaite» des habitants en fonction de leur situation sur le marché du travail. À l'équilibre du modèle, on aboutit à un déséquilibre total dans l'espace où les chômeurs sont relégués à l'extrême périphérie, la fracture spatiale qui apparaît dans le modèle est encore plus forte que celle observée empiriquement. Cela est dû à l'hypothèse d'absence de coût de déménagement qui entraîne un ajustement immédiat de la localisation des agents quand change leur statut sur le marché du travail, mais on peut y voir aussi le résultat de l'absence des politiques publiques qui dans la réalité sont mises en œuvre pour réduire cette fracture. Ces politiques pourraient d'ailleurs être un élément explicatif des différences observées entre les villes américaines et européennes : aux États-Unis, où les politiques publiques seraient moins développées, la ségrégation spatiale semble plus importante qu'en Europe, où l'intervention publique limiterait une ghettoïsation trop marquée.

Nous explorons ci-après les prédictions du modèle quant aux effets des différentes politiques publiques locales envisageables pour réduire le chômage et la fracture spatiale : politique de transport, de formation, de logement social, de densification de l'habitat et fiscalité locale.

\section{Baisser les coûts de transport}

La municipalité peut décider de subventionner les transports, afin de favoriser les déplacements domicile-travail (Zenou, 2000). Elle a alors le choix entre prendre en charge une partie du coût unitaire $t$, ce qui revient à aider l'ensemble des travailleurs, ou donner une subvention ciblée aux plus éloignés des entreprises, en l'occurrence les chômeurs. Une baisse du coût de transport réservée aux chômeurs équivaut à une diminution de la proportion $\alpha$.

Le tableau 2 présente les effets respectifs d'un accroissement de $t$ et de $\alpha$.

Tableau 2 : variation des coûts de transport

\begin{tabular}{|l|c|c|c|c|c|c|}
\hline & $\theta$ & $\lambda$ & $u$ & $R(0)$ & $d^{\prime}$ & $R\left(d^{\prime}\right)$ \\
\hline Hausse $t$ & - & - & + & $?$ & - & + \\
Hausse $\alpha$ & + & + & - & + & + & $?$ \\
\hline
\end{tabular}

\section{Aide généralisée à l'ensemble des habitants}

Une baisse du coût de transport étendue à l'ensemble des habitants (baisse de $t$ ) a un effet très favorable sur le fonctionnement du marché du travail urbain. En effet, il en résulte un accroissement de la tension sur le marché du travail et de l'inadéquation limite. Les travailleurs ont donc plus de facilité pour retrouver un emploi et le chômage diminue.

Comment la baisse du coût de transport rétroagit-elle sur le marché du travail ? Cette baisse correspond à une subvention versée aux travailleurs à hauteur de leur coût de transport. L'aide versée aux salariés est donc plus importante que celle réservée aux chômeurs, puisque ces derniers se déplacent moins. Il en résulte donc une baisse de la différence de coût de localisation entre un salarié et un chômeur $\left(T_{E}-T_{U}\right)$, les salaires négociés seront donc plus faibles et les entreprises plus profitables.

Le chômage baissant, le secteur où les travailleurs privés d'emploi s'installent (à la périphérie de la ville) se contracte. L'effet global sur les loyers du centre-ville reste indéterminé car ils sont soumis à deux effets de sens contraire : la baisse du chômage tend à les faire augmenter, alors que la baisse du coût de transport les réduit en diminuant le bénéfice à habiter près des entreprises.

Malgré ses effets positifs sur la situation de l'emploi, la politique de baisse des coûts de transports n'a cependant pas atteint son but en ce qui concerne la fracture spatiale. Les chômeurs habitent toujours exclusivement à la périphérie de la ville, loin des entreprises, et aucune mixité sociale ne s'est installée.

La seule solution théorique pour que cette politique soit efficace concernant la ségrégation résidentielle serait que la commune prenne en charge l'intégralité des coûts de transport, supprimant ainsi la dimension géographique du modèle et donc la préférence des travailleurs pour la proximité aux emplois. En effet, sans coût de transport, salariés et chômeurs resteraient aléatoirement répartis sur le territoire et donc mélangés, et tous les loyers seraient égaux au prix de location de la terre à l'extérieur de la ville $\left(R_{A}\right)$ étant donnée l'absence de bénéfice à habiter plus près des entreprises. 


\section{Aide ciblée pour les chômeurs}

Alternativement, la municipalité peut décider de ne subventionner que les chômeurs qui sont les agents dont les revenus sont les plus faibles et qui habitent le plus loin du centre-ville. L'effet d'une telle subvention est analytiquement équivalent à celui d'une baisse du nombre de déplacements nécessaires aux chômeurs pour mener à bien leur recherche d'emploi, c'est-à-dire une baisse de $\alpha$. Cependant, dans ce modèle, une baisse du coût de transport supporté par les chômeurs a les mêmes effets négatifs sur le fonctionnement du marché du travail qu'une hausse des allocations-chômage. La subvention améliore les opportunités extérieures des chômeurs, les salaires négociés sont donc plus élevés et la profitabilité des entreprises est plus faible. La tension sur le marché du travail diminue. L'exigence sur la qualité de l'appariement augmente, moins de contacts aboutissent à des embauches. Ces deux effets se combinent pour provoquer la hausse du taux de chômage qui est le coût à payer pour l'amélioration de l'utilité instantanée des travailleurs en recherche d'emploi.

À distance égale, tous les loyers diminuent, et c'est notamment le cas des loyers au centre-ville. Cependant, la séparation géographique entre les deux populations, salariés et chômeurs, n'est pas remise en cause par cette politique d'aide ciblée. En outre, l'allongement de la durée moyenne de chômage accroît les inégalités sociales entre les uns et les autres.

Cette politique, comme la précédente, n'incite donc pas les travailleurs à la mobilité géographique. Elle se limite à un rapprochement « fictif » des chômeurs et des emplois, sans mobilité effective, par le biais d'une politique de "discrimination positive » en faveur des plus éloignés des entreprises.

Des deux politiques envisagées, seule une politique de baisse des coûts de transports bénéficiant à l'ensemble des travailleurs permet de réduire le taux de chômage. En effet, une mesure en faveur des seuls chômeurs, envisageable pour des raisons sociales, a un impact défavorable sur l'emploi. Cependant, ni l'une ni l'autre ne permettent de réduire la fracture spatiale. De ce point de vue, ces politiques aménagent le statu quo sans le changer.

En revanche, si la puissance publique envisageait de subventionner les transports des salariés plutôt que des chômeurs (en dépit de l'iniquité qu'il peut y avoir à aider les plus riches), il serait possible de ramener le coût de transport de ces salariés au même niveau que celui supporté par les chômeurs $(\alpha t d)$. Dans ce cas, le coût de transport de tous les travailleurs serait identique quel que soit leur statut sur le marché du travail, tous seraient alors disposés à payer la même structure de loyers et se répartiraient au hasard sur l'ensemble de l'espace urbain comme dans le cas de transports gratuits. Il n'y aurait plus de fracture spatiale.

\section{Développer la formation}

Comme il existe une inadéquation des qualifications, la puissance publique peut favoriser les appariements par une politique éducative visant à améliorer la qualification des chômeurs d'une façon qui permette de limiter l'effet défavorable de l'inadéquation des qualifications sur la productivité des entreprises. La formation permet donc de rendre les travailleurs plus polyvalents.

Cette politique qui ne concerne à un instant donné que les chômeurs, vise à faciliter leur retour à l'emploi en augmentant le nombre des contacts susceptibles d'aboutir à une embauche. Cependant, comme tous les travailleurs passent par une succession de phases d'emploi et de chômage, on peut considérer, à l'équilibre stationnaire, que tous ont bénéficié de la politique de formation.

L'amélioration de la formation des travailleurs pourrait réduire l'impact de l'inadéquation des qualifications sur la productivité des emplois occupés. On note $\varphi(\varphi \geq 1)$ le paramètre qui mesure l'effet d'une telle politique sur la production. On peut en effet considérer que la fonction d'adéquation s'écrit désormais : $y(\ell / \varphi)$. Une telle politique de formation ne change rien à la productivité d'un travailleur parfaitement adapté à son emploi (soit $\frac{\partial^{2} y}{\partial \ell d \varphi}=0$ pour $\ell=0$ ), mais améliore celle de tous les emplois occupés pour lesquels l'adéquation des qualifications entre l'entreprise et le travailleur n'est pas parfaite (soit $\frac{\partial^{2} y}{\partial \ell d \varphi}>0$ pour $\ell>0$ )).

Tableau 3 : formation des travailleurs

\begin{tabular}{|c|c|c|c|c|c|c|}
\hline & $\theta$ & $\lambda$ & $u$ & $R(0)$ & $d^{\prime}$ & $R\left(d^{\prime}\right)$ \\
\hline Hausse $\varphi$ & + & + & - & + & + & - \\
\hline
\end{tabular}

Une telle politique de formation permet effectivement d'assouplir la contrainte d'adéquation des qualifications de l'entreprise et du travailleur. L'inadéquation limite $\lambda$ s'accrôit donc. Grâce à la formation des travailleurs, cet accroissement ne se fait pas aux dépens de la productivité des entreprises actives. Comme les emplois vacants sont plus rapidement pourvus, davantage d'entreprises vont s'installer dans le bassin d'emploi et la tension sur le marché du travail augmente. Les augmentations de $\lambda$ et de $\theta$ se combinent pour aboutir à une diminution du chômage. 
La régression du chômage se traduit par un accroissement de l'espace occupé par les salariés et la frontière $d$ ' s'éloigne du centre-ville. L'accroissement du nombre de salariés qui supportent un coût de transport important renforce la valeur des terrains situés à proximité des entreprises et les loyers de centre-ville augmentent.

Malgré ses effets positifs en termes d'emploi cette politique de formation des travailleurs ne permet pas de réduire la fracture spatiale. Les chômeurs restent cantonnés aux abords de la ville. Cependant, la durée moyenne du chômage se réduisant, la fracture sociale, fonction du statut sur le marché du travail, est moins forte puisque les travailleurs subissent moins longtemps cette situation défavorable quand ils perdent leur emploi.

\section{Densifier l'habitat}

Pour essayer de réduire la fracture spatiale, la puissance publique peut aussi être tentée de modifier directement les règles qui régissent le fonctionnement du marché immobilier et, par exemple, les règles d'urbanisme. Il est notamment possible d'imaginer une densification de l'habitat, ce qui revient à réduire la surface occupée par chaque habitant.

À population constante, cette densification réduit la taille de la ville, et limite donc les coûts de transport supportés par les travailleurs. Analytiquement, l'effet d'une densification de l'habitat peut être assimilé aux conséquences d'une baisse de $d$.

Tableau 4 : variation de l'espace occupé par un travailleur

\begin{tabular}{|l|c|c|c|c|c|c|}
\hline & $\theta$ & $\lambda$ & $u$ & $R(0)$ & $d^{\prime}$ & $R\left(d^{\prime}\right)$ \\
\hline Hausse $d$ & - & - & + & $?$ & $?$ & + \\
\hline
\end{tabular}

La diminution de la taille de la ville réduit la longueur et le coût des déplacements domiciletravail effectués par les habitants. Les coûts de transport du salarié et du chômeur baissent ainsi proportionnellement à l'usage qu'ils en ont. La situation des salariés s'améliore donc davantage que celle des chômeurs comme lors d'une baisse généralisée du coût de transport. Les effets positifs sur le marché du travail sont donc similaires à ceux d'une baisse de $t$. L'effet global sur les loyers du centre-ville est incertain, car si la baisse de la distance à la périphérie tend à les faire baisser, la diminution du chômage tend au contraire à les faire augmenter.

La situation des travailleurs va donc s'améliorer à condition que la diminution de l'espace occupé par chacun n'affecte pas défavorablement leur utilité. Cependant, pas plus que les politiques précédentes, la densification de l'habitat ne permet de réduire la fracture spatiale : les salariés continuent à habiter près des emplois et les chômeurs restent relégués à la périphérie de la ville. Dès lors, la puissance publique peut être tentée de modifier plus directement la structure de l'espace urbain.

\section{Investir dans le logement social}

Les décideurs publics ont en effet l'opportunité de se substituer sur une partie du territoire à l'offreur sur le marché de l'immobilier pour favoriser l'installation des ménages les plus modestes à proximité des emplois.

Dans cette perspective, la collectivité locale va devenir propriétaire d'une partie du territoire, en achetant de la terre au propriétaire foncier. Elle devient alors second offreur sur le marché de l'immobilier. Supposons qu'elle acquiert au moins une partie des terrains situés à proximité des emplois (terrains situés entre 0 et $d$ ' tel qu'il est défini avant son intervention). Contrairement à l'offreur privé, son critère d'attribution des terrains à la location n'est pas l'offre de loyer la plus élevée, mais un critère de ressources. On fait l'hypothèse, pour simplifier, que l'objectif de la municipalité est de susciter la mobilité "inter-zone", et donc qu'elle se propose de louer ses terres aux seuls chômeurs.

Le loyer proposé par le bailleur public est donc fonction du coût de localisation, $T_{U}$, supporté par les chômeurs. Or le loyer est une fonction décroissante du coût de transport. Plus les chômeurs habitent loin (plus ils assument des coûts de transport élevés), moins ils sont disposés à verser des loyers importants. Si la municipalité propose des logements sociaux à proximité des emplois, les chômeurs qui en bénéficient ont un coût de transport réduit, et sont prêts à payer davantage pour se loger. La tarification équitable des logements sociaux est donc telle que, pour les chômeurs, le supplément loyer demandé compense exactement le gain réalisé en termes de coût de transport. Cette tarification permet un traitement équitable des chômeurs : ceux qui restent à la périphérie paient un loyer moins élevé que ceux qui bénéficient des logements sociaux plus centraux ${ }^{(3)}$.

Dans le même temps, les terrains occupés par les logements sociaux ne peuvent plus l'être par les salariés qui y résidaient précédemment. Ils sont contraints de déménager sur des terrains privés. Comme les salariés continuent de toucher une rémunération supérieure aux allocations-chômage et à avoir des coûts de transports supérieurs à ceux des chômeurs, ils s'installent sur les parcelles contigües au territoire que les salariés occupaient avant la mise en place des logements sociaux. Cette mesure repousse les chômeurs restés à la périphérie plus loin du centre-ville, car la frontière $d$ ' recule à nombre de salariés constant. 
Comme en moyenne les salariés sont contraints de s'installer plus loin des emplois, leur coût de transport moyen augmente, et la concurrence sur le marché privé de l'immobilier induit de ce fait une hausse des loyers au centre-ville.

Le mélange de population n'est donc effectif que pour les chômeurs bénéficiant des logements sociaux, et ce sont leur nombre et leur localisation qui vont déterminer le degré de mixité géographique. La politique de logement social se traduit pas la création ex nihilo d'une mixité. Plus la surface libérée pour les logements sociaux est grande, moins nombreux seront les chômeurs qui resteront cantonnés à la périphérie du bassin d'emploi. Un fractionnement du logement social en différents points de la ville permet alors de mélanger véritablement les deux populations.

Parmi les mesures envisagées jusqu'ici, le développement de logements sociaux en centre-ville constitue le premier dispositif susceptible d'atténuer réellement la fracture spatiale, à hauteur de la surface de terrains dont la puissance publique est capable de se porter acquéreur.

Cette politique peut-elle avoir un effet sur le fonctionnement du marché du travail ? Un tel effet ne peut passer que par une éventuelle modification des coûts de localisation des travailleurs. Même si le bailleur public établit des loyers qui laissent le coût de localisation égal à $T_{U}$ pour l'ensemble des chômeurs, la modification de la structure de l'espace urbain va entraîner un changement concernant celui des salariés dont certains sont relégués plus loin des entreprises.

Soit $\pi$ la part des chômeurs bénéficiant de logements sociaux et vivant donc dans un secteur qui dans d'autres circonstances ne serait occupé que par des salariés. Seuls $(1-\pi) u$ chômeurs habitent encore à la périphérie. Il en résulte que les salariés les plus éloignés du centre-ville habitent à une distance : $d^{\prime}=(1-(1-\pi) u) d$. C'est cet éloignement maximal qui détermine le loyer payé à la distance $d$ ' et le coût de localisation global supporté par les salariés :

$T_{E}=R\left(d^{\prime}\right)+t d^{\prime}=R_{A}+t d[1-(1-\pi)(1-\alpha) u]$

Plus $\pi$ est élevé, plus certains salariés sont conduits à s'installer loin des entreprises, plus le coût de localisation des salariés en général s'accroît, ainsi que les loyers de centre-ville.

Si la différence entre les coûts de localisation des salariés et des chômeurs augmente avec la part des chômeurs bénéficiant de logements sociaux, les salaires négociés par les travailleurs s'accroissent donc également. Il en résulte une baisse de la profitabilité des entreprises qui conduit à une diminution des créations d'emploi.
Parmi les politiques envisagées, la construction de logements sociaux est la première qui soit susceptible d'engendrer une véritable mixité spatiale. Cependant, ses effets sur le marché du travail ne sont pas favorables. Sans doute faudra-t-il envisager de combiner cette politique avec d'autres mesures pour compenser cet inconvénient.

\section{Combiner les politiques publiques et les financer}

Nous avons envisagé aux paragraphes précédents un panel de politiques publiques visant à aider à l'insertion des chômeurs sur le marché du travail, ou à compenser le coût dû à l'éloignement des entreprises par des aides aux transports, ou encore à modifier le fonctionnement du marché de l'immobilier. Toutes les mesures étudiées affectent la répartition des habitants entre chômage et emploi et la productivité moyenne des entreprises. Il semble particulièrement favorable pour la collectivité locale de subventionner les transports de l'ensemble des habitants, de mettre en place une politique de formation visant à réduire les effets défavorables de l'inadéquation des qualifications ou d'agir sur la densité de l'habitat. Cependant, si la limite géographique entre les zones habitées respectivement par les salariés et par les chômeurs s'éloigne du centre-ville du fait de la baisse du chômage, la fracture spatiale entre les uns et les autres n'en est pas réduite pour autant.

En réalité, seule une politique ambitieuse de logements sociaux paraît réellement efficace pour atteindre un objectif de mixité sociale. Etant donné son effet peu favorable sur le niveau du chômage, une telle politique devrait être combinée avec au moins l'un des dispositifs envisagés précédemment.

Se pose cependant la question du financement de ces mesures. Dans notre cadre d'analyse, on peut envisager de prélever un impôt soit sur les entreprises, soit sur les travailleurs. Cependant l'imposition des entreprises réduit leur profitabilité et donc le nombre des implantations d'unités productives dans la ville considérée, ce qui va automatiquement dégrader la situation des travailleurs locaux. Une taxe d'habitation pesant directement sur les ménages semble paradoxalement beaucoup moins défavorable à ces derniers, à condition toutefois de respecter certaines règles. En effet, les travailleurs habitant à l'extrême périphérie (au point $d$ ) ne peuvent pas avoir à supporter une imposition à laquelle ils échapperaient en se déplaçant hors des limites de la ville. Ensuite, on peut montrer que la taxe versée peut être de plus en plus élevée au fur et à mesure que l'on se rapproche du centre-ville sans pour autant inciter les ménages concernés au déménagement (la taxe supportée par un travailleur résidant au point $x$ pourrait par exemple prendre la forme suivante : $\tau(d-x))$. Si un tel système d'imposition ne détériore pas l'utilité des 
travailleurs c'est que, du fait de la concurrence sur le marché de l'immobilier qu'exercent les territoires contigus à la ville, c'est la rente du propriétaire foncier qui va diminuer du montant global de taxe payée par les habitants. Quel que soit $x$ le loyer diminue de la taxe que doit supporter le locataire. Le coût global de localisation de chaque travailleur va donc rester inchangé, et le fonctionnement du marché du travail n'en sera pas affecté.

Cependant, la taxe prélevée ne peut pas représenter un montant supérieur à la différence entre le loyer initial et le loyer à l'extérieur de la ville, $R_{A}$ (voir figure 5).

Les ressources de la collectivité locale restent donc limitées si cette dernière choisit de ne pas taxer les entreprises pour ne pas nuire à l'emploi. Les recettes fiscales ainsi obtenues pourront être partagées entre l'investissement dans les logements sociaux et par exemple la formation des travailleurs. Une répartition adéquate permettra de créer une véritable réduction de la fracture spatiale sans conséquence négative sur l'état du marché du travail.

\section{Figure 5 : taxe d'habitation et rente du propriétaire terrien}

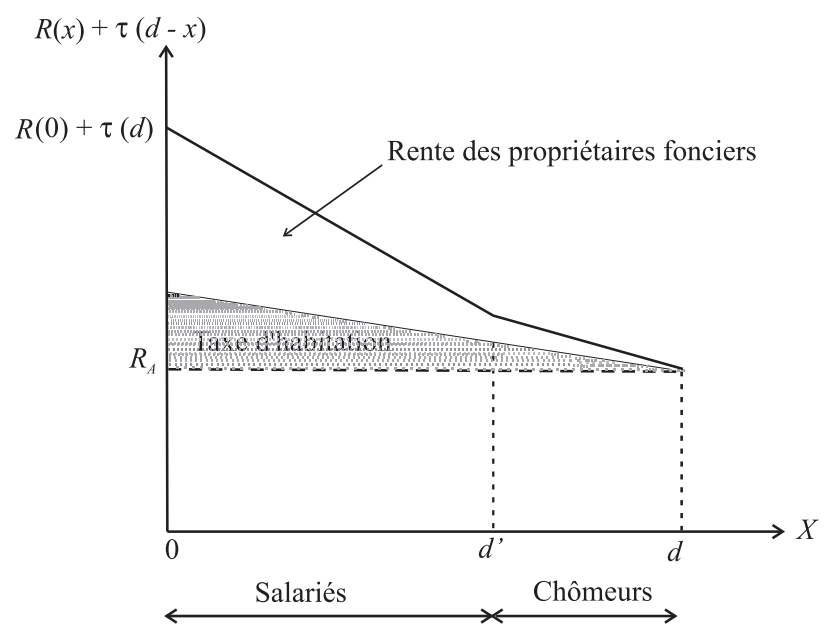

\section{Conclusions}

Le cadre analytique que nous avons développé en combinant un modèle d'appariement avec hétérogénéité des agents et un modèle d'économie urbaine nous a permis d'envisager les effets des différentes politiques qui peuvent être mises en œuvre localement pour réduire le chômage ou la fracture spatiale. Il existe des politiques efficaces pour réduire le chômage, diminuer sa durée et réduire les inégalités sociales, telle qu' une baisse des coûts de transport, le développement des actions de formation ou encore, la densification de l'habitat. Toutefois, ces politiques ne parviennent pas à réduire la fracture spatiale et la ségrégation urbaine. Seule une politique de logement social peut atténuer les inégalités spatiales mais ses effets sur le chômage ne sont pas favorables. C'est pourquoi il importe de combiner les instruments de politique publique pour pouvoir combattre à la fois le chômage et la fracture spatiale. Les possibilités de financement limitent néanmoins les capacités d'intervention des pouvoirs publics et donc l'ampleur des politiques mises en œuvre.

Chaque politique analysée met en évidence les interactions qui existent entre le marché du travail et le marché de l'immobilier. Le modèle pourrait néanmoins être amélioré en prenant en compte au moins deux mécanismes qui renforceraient l'impact de la localisation dans l'espace urbain sur la situation des habitants sur le marché du travail. D'une part, la probabilité pour qu'un chômeur retrouve un emploi pourrait dépendre de la distance qui le sépare des entreprises conformément aux résultats obtenus dans plusieurs études empiriques. Les logements sociaux de centre-ville présenteraient alors un avantage considérable pour leurs occupants relativement aux chômeurs résidant en périphérie, ce qui pourrait poser un problème d'équité sur lequel il conviendrait de s'interroger. D'autre part, on peut raisonnablement penser que la capacité des salariés à conserver leur emploi dépend, pour des raisons d'efficacité, de la distance entre leur lieu de résidence et le centre-ville où se situe l'entreprise qui les emploie. Dans ces conditions, les travailleurs les mieux rémunérés, qui sont aussi les mieux adaptés aux caractéristiques de leur poste, seraient disposés à supporter un loyer, et dans le cas présent un coût global de localisation, plus important que les autres pour se rapprocher de leur entreprise. Le supplément de loyer du centre-ville par rapport à la périphérie dépasserait alors les coûts de transport économisés et le salaire deviendrait le déterminant direct du lieu de résidence. L'introduction de tels mécanismes constituent quelques-unes des pistes qu'il conviendrait d'explorer dans le cadre de futures recherches. 


\section{Notes}

(1) La maximisation des utilités intertemporelles et la minimisation des coûts de localisation sont équivalentes sur le plan analytique, car aucune autre variable des équations (5) et (6) ne dépend de la distance du lieu d'habitation au centre.

(2) Sauf à supposer comme le font Wasmer et Zenou (2006) qu'une plus grande proximité des entreprises améliore directement la probabilité des chômeurs de trouver un emploi.

(3) Cependant, si dans notre modèle l'efficacité de la recherche d'emploi était fonction de la distance géographique, l'accès aux logements sociaux donnerait un avantage aux bénéficiaires sur le marché du travail.

\section{Bibliographie}

Brueckner J. et Martin R. (1997). "Spatial Mismatch: An Equilibrium Analysis", Regional Science and Urban Economics, vol. 27, n 6, pp. 693-714.

Cavaco S. et Lesueur J.Y. (2004). "Contraintes spatiales et durée de chômage", Revue Française d'Économie, vol. 18, $\mathrm{n}^{\circ} 3$, pp. 229-257.

Fujita M. (1989). Urban Economic Theory, Cambridge University Press, Cambridge.

Gavrel F. et Lebon I. (2008). "Salaire minimum, allocations chômage et efficacité du marché du travail", Recherches économiques de Louvain 2008/1, vol. 74, n 1, pp. 53-75.

Hellerstein J., Neumark D. et McInerney M. (2008). "Changes in Workplace Segregation in the United States between 1990 and 2000: Evidence from Matched Employer-Employee Data", NBER Chapters, in: The Analysis of Firms and Employees: Quantitative and Qualitative Approaches, pp. 163-195, National Bureau of Economic Research, Inc, University of Chicago Press.

Immergluk D. (1998). "Job Proximity and the Urban Employment Problem: Do Suitable Nearby Jobs Improve Neighbourhood Employment Rates?", Urban Studies, vol. 35, $\mathrm{n}^{\circ} 1$, pp. 7-23.

Kain J. (1968). "Housing Segregation, Negro Employment, and Metropolitan Decentralization", Quarterly Journal of Economics, vol. 82, n 2 , pp. 175-197.

Marimon R. et Zilibotti F. (1999). "Unemployment vs. Mismatch of Talents: Reconsidering Unemployment Benefits", Economic Journal, vol. 109, n 455, pp. 266-291.

O'Regan K.M. (1993). "The Effect of Social Networks and Concentrated Poverty on Black and Hispanic Youth Unemployment", vol. 27, n 4, pp. 327-342.

Pissarides C. (2000). Equilibrium Unemployment Theory, $2^{\mathrm{e}}$ ed., MIT Press.

Rogers C. (1997). "Job Search and Unemployment Duration: Implications for the Spatial Mismatch Hypothesis", Journal of Urban Economics, vol. 42, n 1, pp. 109-132.

Wasmer E. et Zenou Y. (2006). "Equilibrium Search Unemployment with Explicit Spatial Frictions", Labour Economics, vol. 13, n 2, pp. 143-165.

Zenou Y. (2000). "Urban Unemployment, Agglomeration and Transportation Policies", Journal of Public Economics, vol. $77, \mathrm{n}^{\circ} 1$, pp. 97-133. 